\title{
Erratum to: Social support and suicidal ideation in Japan: are home visits by commissioned welfare volunteers associated with a lower risk of suicidal ideation among elderly people in the community?
}

\author{
Masayuki Noguchi • Toshihide Iwase • \\ Etsuji Suzuki · Yoko Kishimoto · Soshi Takao
}

Published online: 12 October 2014

(C) Springer-Verlag Berlin Heidelberg 2014

Erratum to: Soc Psychiatry Psychiatr Epidemiol (2014)

49:619-627

DOI 10.1007/s00127-013-0752-5

In the original publication of the article, the following errors have occurred. In Table 1, the labels "No" and "Yes" of the first six variables to assess social support (SS1 to SS6), and "Not living together" and "Living together" of the characteristic "Marital status" have been reversed inadvertently. In Table 2, the mean of "Social support (SS1-7)" among men should be 5.80 instead of 5.79. In the fourth line from the bottom on page 624 , the sentence "Third, they numbered about 28,000 " should read, "Third, they numbered about 23,000". Also, in the third line from the bottom of page 624, "This is more than 30 times" should read, "This is more than 20 times".

The online version of the original article can be found under doi: 10.1007/s00127-013-0752-5.

M. Noguchi $(\bowtie)$

Okayama Prefectural Mental Health and Welfare Center,

1-1-10-101 Furugyo-cho, Naka-ku, Okayama 703-8278, Japan

e-mail: nogumasa917@gmail.com

T. Iwase

Support Center for Medical Cooperation, Human Resource Placement, and Career Promotion of Okayama Prefecture, Graduate School of Medicine, Dentistry and Pharmaceutical

Sciences, Okayama University, Okayama, Japan

E. Suzuki $\cdot$ Y. Kishimoto $\cdot$ S. Takao

Department of Epidemiology, Graduate School of Medicine, Dentistry and Pharmaceutical Sciences, Okayama University,

Okayama, Japan 
Table 1 Characteristics of the study subjects, Okayama, Japan, 2010

$S D$ standard deviation

a Assessed with the Instrumental Activities of Daily Living Scale by Lawton and Brody. Summary scores were obtained by adding IADLs 1,2 , 6,7 , and 8 for men, and IADLs 1-8 for women

\begin{tabular}{|c|c|c|c|}
\hline Characteristics & Total $(N=11,218)$ & $\operatorname{Men}(N=4,525)$ & Women $(N=6,693)$ \\
\hline Mean age, year (SD) & $76.7(7.3)$ & $76.0(6.9)$ & $77.1(7.5)$ \\
\hline \multicolumn{4}{|l|}{ Suicidal ideation, $n(\%)$} \\
\hline Yes & $1,124(10.0)$ & $360(8.0)$ & $764(11.4)$ \\
\hline No & $10,094(90.0)$ & $4,165(92.0)$ & $5,929(88.6)$ \\
\hline \multicolumn{4}{|l|}{ Social support (SS1-7) } \\
\hline \multicolumn{4}{|l|}{ Shopping (SS1), $n(\%)$} \\
\hline No & $1,320(11.8)$ & $538(11.9)$ & $782(11.7)$ \\
\hline Yes & $9,898(88.2)$ & $3,987(88.1)$ & $5,911(88.3)$ \\
\hline \multicolumn{4}{|c|}{ Cleaning and cooking (SS2), $n(\%)$} \\
\hline No & 2,011 (17.9) & $603(13.3)$ & $1,408(21.0)$ \\
\hline Yes & $9,207(82.1)$ & $3,922(86.7)$ & $5,285(79.0)$ \\
\hline \multicolumn{4}{|c|}{ Offer to run errands (SS3), $n(\%)$} \\
\hline No & $1,697(15.1)$ & $636(14.1)$ & $1,061(15.9)$ \\
\hline Yes & $9,521(84.9)$ & $3,889(85.9)$ & $5,632(84.2)$ \\
\hline \multicolumn{4}{|l|}{ Reassure (SS4), $n(\%)$} \\
\hline No & $1,647(14.7)$ & $709(15.7)$ & $938(14.0)$ \\
\hline Yes & $9,571(85.3)$ & $3,816(84.3)$ & $5,755(86.0)$ \\
\hline \multicolumn{4}{|l|}{ Be on the side of (SS5), $n(\%)$} \\
\hline No & $1,986(17.7)$ & $672(14.9)$ & $1,314(19.6)$ \\
\hline Yes & $9,232(82.3)$ & $3,853(85.2)$ & $5,379(80.4)$ \\
\hline \multicolumn{4}{|l|}{ Take care of (SS6), $n(\%)$} \\
\hline No & $1,397(12.5)$ & $533(11.8)$ & 864 (12.9) \\
\hline Yes & $9,821(87.6)$ & $3,992(88.2)$ & $5,829(87.1)$ \\
\hline \multicolumn{4}{|c|}{ Home visits by commissioned welfare volunteers (SS7), $n(\%)$} \\
\hline No & $4,061(36.2)$ & $1,757(38.8)$ & $2,304(34.4)$ \\
\hline Yes & $7,157(63.8)$ & $2,768(61.2)$ & $4,389(65.6)$ \\
\hline \multicolumn{4}{|l|}{ Educational attainment, $n(\%)$} \\
\hline Junior high school or lower & 4,992 (47.1) & $2,108(48.4)$ & $2,884(46.1)$ \\
\hline High school & $4,615(43.5)$ & $1,782(40.1)$ & $2,833(45.3)$ \\
\hline College or higher & $996(9.4)$ & $463(10.6)$ & $533(8.5)$ \\
\hline Missing & 615 & 172 & 443 \\
\hline \multicolumn{4}{|l|}{ Marital status, $n(\%)$} \\
\hline Not living together & $4,324(38.6)$ & 888 (19.6) & $3,436(51.3)$ \\
\hline Living together & $6,894(61.5)$ & $3,637(80.4)$ & $3,257(48.7)$ \\
\hline \multicolumn{4}{|c|}{ Instrumental activities of daily living ${ }^{\mathrm{a}}, n(\%)$} \\
\hline Mean scores (SD) & & $4.33(1.26)$ & $6.72(2.19)$ \\
\hline Missing & & 748 & 2,427 \\
\hline
\end{tabular}

Acknowledgments The authors thank Yuki Otsuka of Okayama University, Okayama, Japan for bringing some of these errors to their attention. 